\title{
Exploration of user experience innovation on the application of detecting blood glucose
}

\author{
Yonghai $\mathrm{Yu}^{1, \mathrm{a}}$, Huangyan $\mathrm{Qi}^{1, \mathrm{~b}}$ and Jinsong Zhang ${ }^{1, \mathrm{c}}$ \\ ${ }^{1}$ Zhejiang University of Technology 310023 Zhejiang, China; \\ a382249707@qq.com, bhzQihuangyan@163.com, ' $132197 @ q q . c o m$
}

Keywords: Blood Glucose Testing, User Experience Innovation, emotional.

\begin{abstract}
Giving users the convenience of life, the blood glucose monitoring apps are very popular among diabetics. In this paper, on the basis of experience analysis of the application of existing blood glucose monitoring apps and the demand analysis of such apps, the user experience goals of glucose monitoring apps were concluded. At the same time, according to the experience goals, the innovative thinking of creating "emotional” blood glucose monitoring apps was put forward.
\end{abstract}

\section{Preface}

As people's living standard is increasingly raised, diabetes has become a common chronic disease which threatens residents' physical health. Diabetes patients are likely to have negative emotions such as loneliness, helplessness and lack of sense of safety. At this time, they have urgent demand for comfort so they hope to be cared by families, the society and doctors, etc. At present, most of blood glucose monitoring apps focus on blood glucose monitoring and ignore patients' emotional demand. Under such circumstance, the article proposed to develop blood glucose monitoring apps centering on emotional services from the perspective of user experience.

\section{Status Quo of Blood Glucose Monitoring Apps}

Blood glucose value occupies an important role in both diabetes prevention and treatment. Accordingly, persistence in self blood glucose monitoring is of great significance to blood glucose control of diabetes patients. On the one hand, it can help patients grasp blood glucose control status; on the other hand, it can help patients adjust lifestyle and reduce occurrences of hypoglycemia. Blood glucose monitoring apps refer to mobile application and varied smart blood glucose monitoring equipment which realize real-time collection and dynamic analysis of blood glucose data through Bluetooth, WIFI, RFID and other sensor technologies. Mobile apps update, assess and store users' health information by interacting with cloud servers. Blood glucose monitoring apps can be used at home, thus having characteristics of great convenience and low cost while meeting with users' demands.

According to surveys, user experience of current blood glucose monitoring apps mainly includes four aspects including blood glucose collection and analysis experience, data management experience, blood glucose health news service and doctor service experience. In terms of blood glucose collection and analysis experience, most of current apps follow conventional procedures and try to guide users to complete collection in an efficient and quick way. In terms of dynamic analysis, most of apps show graphic results in the way of value and icon combination and provide corresponding suggestions on health. Regards to data management experience, multiple demonstration ways are adopted such as table, trend chart, time flow and pie chart so as to make blood glucose data more vivid and easier to understand. As to blood glucose health news service, some apps try to provide nutrition recipes and science knowledge related with diabetes to strengthen users' cognition of diabetes. As to the last one, most apps attempt to insert the role of doctor to realize long-distance inquiry and communication between patients and doctors, thus simplifying doctor-seeing procedures; and some apps add a QA function to help answer patients' health questions. 
Currently, in the internet era of user experience first, current blood glucose monitoring apps aim to bring better product experience to users from different perspectives but there are some shortcomings: first, in terms of equipment operation guidance, some apps fail to present simple and clear interface design for users, which increases users' learning cost. Second, in data management, most of apps try to use three to four kinds of data demonstration forms. However, there are too much data on some pages, thus causing visual burden to users. Lastly, experience in doctor service is still immature. Some apps have doctor inquiry and diagnosis platforms but the role of doctors is not that apparent. Moreover, it takes a long time to get reply and doctors' reply rate is low, etc. which all reduce users' satisfaction degree with apps.

\section{Demand Analysis of Blood Glucose Monitoring Apps Users}

On the basis of exploring the status quo of current blood glucose monitoring apps, we analyzed target users of such apps and their concrete demands for blood glucose monitoring apps from the perspective of target users.

\subsection{Analysis of Blood Glucose Monitoring Apps Users.}

In daily life, users of blood glucose meter are usually diabetes patients or recessive patients (the aged, obese patients and those with a genetic history). Due to different diabetes categories, patients may be young people less than 30 years old or old people. If users are old people, we need to consider what's special about them, such as slow action and inability to use smart equipment proficiently.

\subsection{Analysis of Demands for Blood Glucose Monitoring Apps.}

According to user analysis above and Maslow's hierarchy of needs, we analyzed diabetes patients' concrete demands for blood glucose monitoring apps from their perspective. Maslow's hierarchy of needs is one of behavioral science theories proposed by American psychologist Abraham Maslow in his paper of “Human Incentive Theory” in 1943. According to them, people's needs are divided into physiological, safety, love/belonging, esteem and self-actualization needs at a hierarchical level [1]. Generally speaking, people will move to a higher level after lower needs are met. Hence, a product with desirable user experience need to bring higher level of product experience while satisfying users' basic needs.

Accordingly, we conclude concrete demands of diabetes patients for blood glucose monitoring apps as follows:

1. Basic needs-health and safety needs. Health is the most basic element for a living person so patients pay greatest attention to their diseases. Yet, for diabetes users, the direct purpose of using blood glucose monitoring apps is to learn about their blood glucose condition and what measures they should take to alleviate the disease. When patients are in the hospital, after checking, doctors will tell them the results. If data is abnormal but within controllable scope, doctors will tell them to have another check after several days; if it's beyond the normal scope, doctors will tell patients to take medicine or be hospitalized. Yet, when patients use mobile apps, they are not in the hospital so mobile apps need to replace doctors and help users analyze diseases and offer scientific suggestions.

2. Social needs (love and belonging). From the perspective of sociology, patients are a special type of social role and they are more sensitive emotionally. They hope to be loved by families, accepted and cared by the society in order to alleviate their anxiety. They hope to have a sense of belonging and mitigate their sense of helpless and loneliness. Social needs can help users find their value of existence. Patients' realization of their social needs is conducive to their disease improvement and psychological health realization.

\subsection{Goals of Experience Innovation in Blood Glucose Monitoring Apps.}

We concluded concrete needs of target users from health and safety need and social need aspects. In the end, we defined user experience goals of blood glucose monitoring apps from three perspectives of instinct, behavior and introspection put forward by Donald .A. Norman.

1. Health and safety needs-blood glucose monitoring experience. In terms of blood glucose monitoring experience, important feedback on users' health should be given the instinct level, including vivid monitoring results, what users need to do next such as timely re-check if it's not 
severe and seeing a doctor when it's severe; at the behavior level, a complete and easy-to-operate procedure should be offered to users. At the introspection level, it should enable users who use it for a long time to establish a sense of safety and trust.

2. Social needs-Family care/patient communication experience. At the instinct level, it should meet with patients and families' demand for real-time communication; in terms of introspection, it should make users feel being respected and have a sense of belonging in the communication process, and bring optimistic emotional experience to users.

\section{Innovative Thoughts of "Emotional” Blood Glucose Monitoring Apps}

According to user experience innovation goals, we proposed the innovative thought of building 'emotional' blood glucose monitoring apps. The following aspects should be emphasized to enhance user experience:

1. Smart blood glucose monitoring experience. Smart blood glucose monitoring experience includes two aspects: smart diagnosis and smart alert. In smart diagnosis, the system will offer scientific diagnosis suggestions according to factors such as users' recent blood glucose value changes, age and types of diabetes while giving results. For instance, if a user's blood glucose value is a bit high but within the controllable scope, the app will remind users to increase test frequency, pay attention to diet and work out, etc. If a user's blood glucose value exceeds the controllable scope, it will prompt users to see a doctor immediately. If users' test results are normal, it will remind users to keep it up, etc. Smart alert means that the system will automatically set test alerts according to users' results. If users have abnormal data recently, the alert frequency will be increased. If users' data is normal, alert frequency will be decreased.

2. Social experience-family interaction experience and patient interaction experience. In terms family interaction experience, children can like their parents, get parents' authorization and view blood glucose monitoring reports through SMS, e-mail or system alert first time after parents finish testing. Patient interaction experience includes two goals in design. First, establishment of patient community. In this way, patients can find their peers while measuring blood glucose, thus having a sense of belonging. Besides, incentive mechanism including credits accumulation is adopted to encourage users to leave a message, let users find their own value and increase their sense of achievement. Second, friend making experience. Users can add friends or join in a group in the system to encourage users to communicate with each other.

3. Other details. Considering that target users may be old people, design such as font and color will be consistent with old people's psychological features as much as possible. Plain words will be used rather than elusive medical terminology; clear and explicit measurement results will be given. Some cue words will be used to guide users on the basis of predicting problems which users may encounter, which can strengthen users' trust for apps. For example, detailed steps of test procedures and key points of wearing can be reminded; under emergencies, some indicative designs can be made. For instance, a relevant icon can flicker when the battery is low; some alerts can be designed for disconnection of hardware and mobile phone.

\section{Conclusion}

The paper started with Maslow's hierarchy of needs, analyzed concrete demands of users for smart blood glucose meter and proposed to build blood glucose monitoring apps centering on emotional service while meeting with basic demands. Besides, it paid attention to users' emotional demands and applied them in actual design, which can not only enhance users' adhesiveness but also provide new thoughts for developing mobile monitoring apps.

With development of mobile internet, more and more mid-age and old-age people are willing to have access to and use the internet, thus laying a solid user foundation for development of mobile medical treatment. It's believed that in near future, mobile medical treatment will embrace its 
prosperity. In the internet era when user experience is deemed as life, better products can be designed only when users' demands are truly grasped from the perspective of user experience.

\section{References}

[1]. H.Maslow Abraham. Motivation and personality [M]. Jinsheng $\mathrm{Xu}$ translation. Renmin University of China press, 2007.4.

[2]. Donald A Norman. Emotional design[M]. He Xiaomei, Qiuxing Ou translation. Beijing: CITIC publishing house, 2005.5.

[3]. Zhang Hehua. Analysis of the health needs of mobile medical [J]. Journal of medical informatics, 2015.05.

[4]. Bruno M.C. Silva. Mobile-health: A review of current state in 2015[J]. Journal of Biomedical Informatics, 56(2015)265-272.

[5]. Xinlei Wang. Mobile medical treatment under the construction of Internet of things[J]. China Medical Herald, 2012.10. 